\title{
Collision Rate Constants for Polarizable Ions
}

\author{
Brian R. Eichelberger, Theodore P. Snow, and Veronica M. Bierbaum \\ Department of Chemistry and Biochemistry, Center for Astrophysics and Space Astronomy, \\ University of Colorado, Boulder, Colorado, USA
}

\begin{abstract}
Langevin described a model for the interaction between an ion and a neutral nearly a century ago and since then, many modifications have been introduced to adjust for specific circumstances. This work discusses the induced dipole-induced dipole interaction between an ion and a neutral without a permanent dipole and introduces an anisotropic adjustment. A point polarizable ion model (PPI) and an orientation dependent polarizable ion model (ODPI) are discussed and applied to systems where the ion is highly polarizable and the neutral is only weakly polarizable. Significant deviations from classical Langevin rate constants and significant differences between PPI and ODPI are observed. (J Am Soc Mass Spectrom 2003, 14, 501-505) @ 2003 American Society for Mass Spectrometry
\end{abstract}

$\mathrm{O}$ ver the last century, many scientists have discussed the collisional rate of an ion with a polarizable neutral. Langevin began the discussion in 1905 [1] with work outlining the attractive potential between a point charge and a point-polarizable neutral. The result was an expression for the collisional rate constant, $\mathrm{k}_{\mathrm{L}}$, in terms of a few simple variables; $e$, the elementary charge, $\alpha_{n}$, the polarizability of the neutral, and $\mu$, the reduced mass of the ionneutral pair.

$$
k_{L}=2 \pi e\left(\frac{\alpha_{n}}{\mu}\right)^{1 / 2}
$$

The approach is appealing in its simplicity, and has been used as a standard for ion-molecule and ion-atom collisions. This method has been used extensively to calculate collision rate constants in many environments, including the interstellar medium, where atomic hydrogen, molecular hydrogen, and atomic oxygen and nitrogen are the most abundant species.

Another major area of work involves the interaction of an ion and a neutral species with a permanent dipole moment. Several approaches have been taken, ranging from an orientation-fixed dipole approximation to orientation-dependent approaches which rigorously conserve angular momentum and energy. These methods have been discussed in detail [2,3]. Additional work in this area involves parameterizing the theory to obtain a

Published online April 3, 2003

The authors dedicate this work to Professor Charles DePuy on the occasion of his 75th birthday, in recognition of his outstanding contributions to ion chemistry as well as his guidance and friendship throughout the years.

Address reprint requests to Dr. V. M. Bierbaum, Department of Chemistry and Biochemistry, University of Colorado, Campus Box 215, Boulder, CO 80309-0215, USA. E-mail: veronica.bierbaum@colorado.edu solution that is easily accessible to experimentalists [4, 5].

Deviations from a point charge model have been described by Su et al. $[9,10]$ and an induced dipoleinduced dipole adjustment has been discussed by $\mathrm{Su}$ and Bowers [11]. The possibility for large deviation is mentioned with respect to anionic systems, but due to the difficulty of acquiring ionic polarizabilities, only the $\mathrm{Cl}^{-}$and $\mathrm{H}^{-}$anions are discussed. These systems afford less than $5 \%$ deviation from the classical Langevin rate constants. In this paper we highlight the importance of the anisotropy of the induced dipole-induced dipole interaction and introduce an orientation dependent polarizable ion model. We demonstrate that these interactions, especially when anisotropy is included, have significant impact on the collision rate constants of large molecular cations and anions.

\section{Theory}

The Langevin rate constant is derived from a potential that assumes the ion is a point charge. Two factors, the long-range nature of the potential involving ions and the way in which the random orientation of an ion causes the location of the charge to average to the center, make this approximation accurate in most cases. Deviation can occur, however, in the second of these two factors. If the charge is mobile within the ion, the average location of the charge can shift from the center, effectively increasing the critical impact parameter.

Traditionally, positive ions have been studied more frequently in laboratory experiments because of the wide range of their applicability and the relative ease with which they are made. In cation systems, ionic polarizability effects have not been observed in experiments where measured rate constants are compared 
with theoretical collision rate constants. For negative ions that are similar in size to these cations, however, one would expect the mobility of the charge to be much larger.

The approach taken to assess the dependence of the collision rate on the polarizability of the ion was to adjust the Langevin potential in the following way:

$$
\begin{aligned}
& V_{\text {eff }}=V_{\text {Langevin }}+V_{\alpha} \\
& V_{\text {Langevin }}=E\left(\frac{b^{2}}{r^{2}}\right)-\frac{1 \alpha_{n} e^{2}}{2 r^{4}}
\end{aligned}
$$

Here, $\mathrm{E}$ is the relative kinetic energy, $\mathrm{b}$ the impact parameter, $r$ the distance between the ion and the neutral, and $\alpha_{\mathrm{n}}$ is the polarizability of the neutral. $\mathrm{V}_{\alpha}$ is an additional term based on the attraction between a polarizable ion and a polarizable neutral.

The approach taken by Su et al. [9] assumes that the ion is a single massive point with a uniform polarizability. The $\mathrm{V}_{\alpha}$ for a point-polarizable ion (PPI)-neutral pair takes the form [11]:

$$
V_{P P I}(r)=-\frac{3}{2} a_{0}{ }^{1 / 2} e^{2} \frac{\alpha_{i o n} \alpha_{n}}{\left(\left(\frac{\alpha_{i o n}}{N_{i o n}}\right)^{1 / 2}+\left(\frac{\alpha_{n}}{N_{n}}\right)^{1 / 2}\right) r^{6}}
$$

where $\mathrm{a}_{0}$ is the Bohr radius, $\alpha_{\text {ion }}$ and $\alpha_{\mathrm{n}}$ are the polarizabilities of the ion and the neutral, respectively. $\mathrm{N}_{\text {ion }}$ and $\mathrm{N}_{\mathrm{n}}$ are the outer shell electrons of an atomic species or the number of electrons in the HOMO of molecular species. The $\mathrm{r}^{-6}$ dependence is typical of the attraction between two polarizable species.

We incorporate an adjustment to this approach, an orientation dependent polarizable ion (ODPI) model, which considers the ion as a tube of charge with an average orientation towards the approaching neutral. This model is useful when the polarizability along one axis differs greatly from the polarizability along the other two. In the longer of the negatively charged and highly conjugated chains we will discuss, the polarizability parallel to the chain is much larger than the polarizability perpendicular to the chain. Two polarizability values are needed, $\alpha_{\perp}$ and $\alpha_{\|}$. When the neutral approaches the chain at an angle $\theta$ (Figure 1), the following expression represents the potential $\mathrm{V}_{\alpha}$ :

$$
\begin{aligned}
& V_{O D P I}(r)= \\
& -\frac{3}{2} a_{0}{ }^{1 / 2} e^{2} \frac{\left(\alpha_{\|} \cos \theta+\alpha_{\perp} \sin \theta\right) \alpha_{n}}{\left(\left(\frac{\left(\alpha_{\|} \cos \theta+\alpha_{\perp} \sin \theta\right)}{N_{\text {ion }}}\right)^{1 / 2}+\left(\frac{\alpha_{n}}{N_{n}}\right)^{1 / 2}\right) r^{6}}
\end{aligned}
$$

In Eq 4, an approach similar to the one taken in ADO theory is utilized to acquire the average value for $\theta$. The

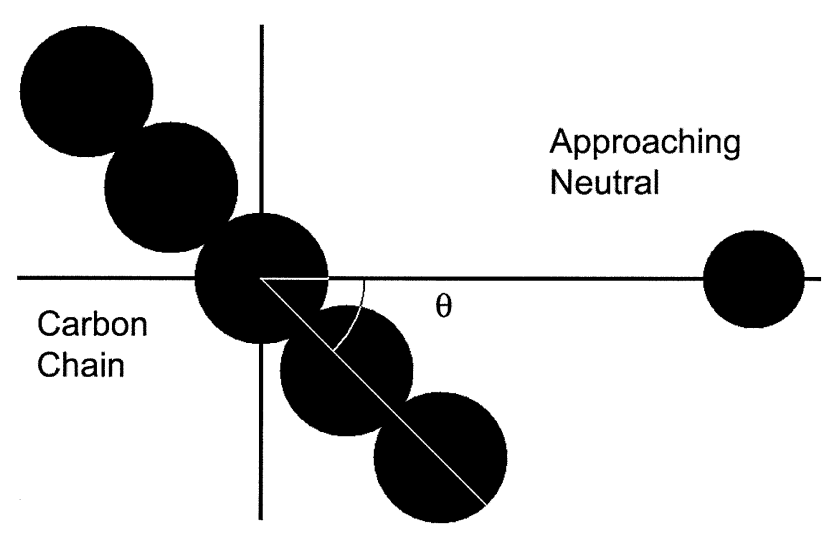

Figure 1. $\theta$ is defined as the angle between the primary axis of the molecule and the approach vector of the neutral.

general expression for the average value of $\theta$ is:

$$
\langle\theta\rangle=\frac{\int_{0}^{\pi / 2} \theta P(\theta) d \theta}{\int_{0}^{\pi / 2} P(\theta) d \theta}
$$

The probability function for $\theta, \mathrm{P}(\theta)$, takes the form,

$$
P(\theta)=\frac{\sin \theta}{\left(E_{r o t}+V_{\alpha}\right)^{1 / 2}}
$$

as described in $\mathrm{ADO}$ theory, where $\mathrm{E}_{\text {rot }}$ is the rotational energy. Due to the $\mathrm{r}^{-6}$ dependence of $\mathrm{V}_{\alpha}$, however, $\mathrm{E}_{\text {rot }}$ $\gg V_{\alpha}$ at room temperature. Since $E_{\text {rot }}$ is independent of $\theta$, Eq 5 reduces to:

$$
\langle\theta\rangle=\frac{\int_{0}^{\pi / 2} \theta \sin \theta d \theta}{\int_{0}^{\pi / 2} \sin \theta d \theta}=1 \text { radian }
$$

ODPI addresses the case where there will be substantial deviation from PPI when considering the polarizabilities along the axes. In other cases, PPI is sufficient.

In both cases, the derivation of the rate constant through the critical impact parameter does not afford a simple expression in terms of a few variables, as with the traditional Langevin theory. Values for $\mathrm{E}$ and the various $\alpha^{\prime}$ s and N's were substituted such that:

$$
V(r)=x b^{2} r^{-2}-y r^{-4}-z r^{-6}
$$

where $x, y$, and $z$ are real, positive numbers. The derivative of $\mathrm{V}$ with respect to $\mathrm{r}$ was then taken and set to zero to calculate $r^{*}(b)$, the value of $r$ in terms of $b$, where $\mathrm{V}$ is at a maximum. Substituting this result into 
Table 1. Values of $k_{L}$ and $k_{P P I}$ for the reaction of $C_{n}^{-}$and $C_{n} H^{-}$with $\mathrm{O}$, the percent increase in the collisional rate constant, and the reaction efficiencies for both theories.

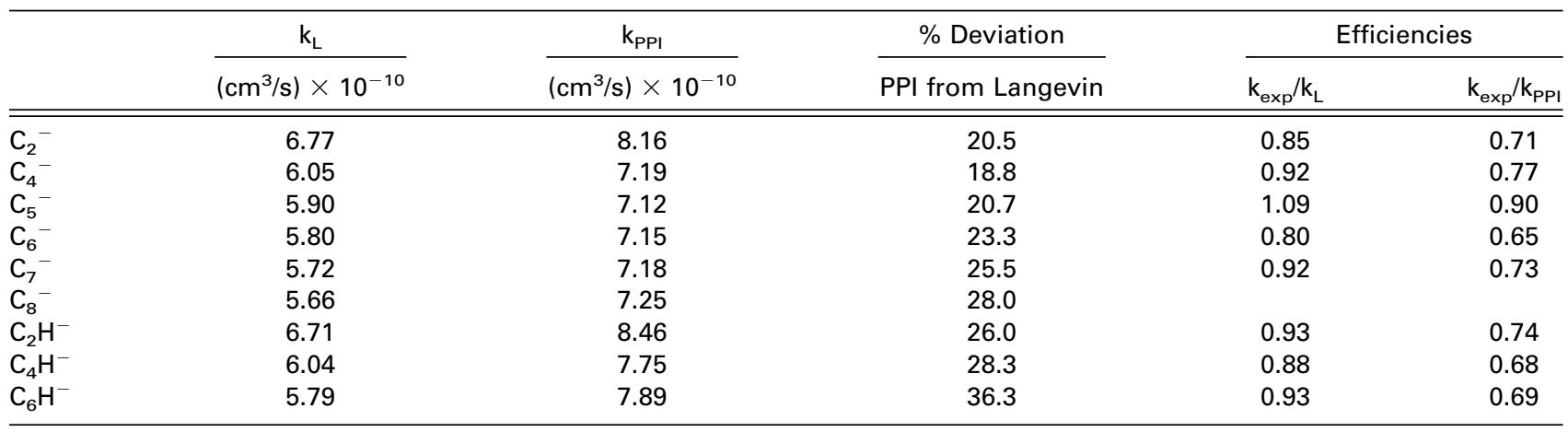

$\mathrm{V}(\mathrm{r})$, we obtain $\mathrm{V}^{*}(\mathrm{~b})$. Now taking $\mathrm{V}^{*}$ as the value for the barrier to collision, E must satisfy the condition $\mathrm{E} \geq$ $\mathrm{V}^{*}$. Setting $\mathrm{E}=\mathrm{V}^{*}(\mathrm{~b})$, a value for $\mathrm{b}^{*}$ can be obtained, where $b^{*}$ is the maximum impact parameter that results in a collision. Given this result, k was found using:

$$
k=\left(\frac{2 E}{\mu}\right)^{1 / 2} \pi b^{* 2}
$$

\section{Results and Discussion}

Because of their potential relevance in the interstellar medium, we have carried out comprehensive experimental studies of the reactions of aromatic cations and of carbon chain anions with $\mathrm{H}_{2}, \mathrm{H}, \mathrm{N}$, and $\mathrm{O}$ [12-17]. In these unusual systems, the polarizability of the neutral is small and that of the ion is large, which accentuates the effect of the $V_{\alpha}$ term on the overall potential. To illustrate this, we have calculated the collisional rate constants for $\mathrm{C}_{\mathrm{n}}^{-}+\mathrm{O}$ and $\mathrm{C}_{\mathrm{n}} \mathrm{H}^{-}+\mathrm{O}$ systems using Langevin theory, PPI and ODPI. [Ionic polarizability values were obtained from Gaussian 98 calculations at the B3LYP/aug-cc-pVDZ level [6-8]. This basis set gave the best polarizability value for neutral benzene and was necessary to obtain minimized structures for the $\mathrm{C}_{\mathrm{n}}^{-}$and $\mathrm{C}_{\mathrm{n}} \mathrm{H}^{-}$anions.] Table 1 shows the results of the PPI approach, where significant deviations from Langevin theory are evident, ranging from $\sim 19-36 \%$.

While the PPI approach affords significant deviation, of specific interest to the reactions of $\mathrm{C}_{n}^{-}$is the ODPI form of $\mathrm{V}_{\alpha}$. For $\mathrm{C}_{2}^{-}, \alpha_{\|}$is less than $\alpha_{\perp}$, but in all longer chains, $\alpha_{\|}$is much larger than $\alpha_{\perp}$ (by more than a factor of 7 in the case of $C_{8}^{-}$). Because of this, we expect there to be a difference between the PPI results and the ODPI results. Figure 2 shows a plot of the potential, $\mathrm{V}_{\alpha}$, as a function of the angle $\theta$ for $\mathrm{C}_{6} \mathrm{H}^{-}+\mathrm{O}$. At the average angle calculated, the potential has a greater value and therefore leads to a larger rate constant.

We expect this difference to increase as the chain length increases. As shown in Table 2, this is the case. With $\mathrm{C}_{2}^{-}$, the change in deviation is less than $1 \%$ but as chain length increases, the change in deviation increases significantly and is greater than $7 \%$ in the longer chains.
This result illustrates that, for small ions, the PPI approximation gives reasonable results, but as the size of the ion increases, consideration of the unique orientation aspects of the system is essential.

The contribution of size to the collisional rate constant has been discussed previously by Su et al. [10]. In their work, an adjustment is added when the size of the ion is larger than the theoretical collisional cross section. In our systems, the impact parameter is defined with respect to the center of mass of each species. The largest of our molecules, $\mathrm{C}_{8}^{-}$, has a distance from the center of mass to the end of the chain of $\sim 4.55 \AA$. The impact parameter for this molecule is $5.6 \AA$ for PPI and $5.7 \AA$ for ODPI. When considering the atomic radii of oxygen, which is $\sim 0.75 \AA$, it is clear that the ion-atom collisional cross section is larger than the rotationally averaged hard sphere collisional cross section for these systems. For this reason, the size consideration previously discussed by $\mathrm{Su}$ et al. it is not necessary in this case.

The $\mathrm{C}_{\mathrm{n}}^{-}+\mathrm{O}$ system has two factors that accentuate the induced dipole-induced dipole interaction. The first is the large polarizabilities of the anions, as discussed above. The second factor is the low polarizability of $\mathrm{O}$ atom. Because the Langevin rate constant depends directly on $\alpha_{\mathrm{n}}$, the question arises, for diverse systems,

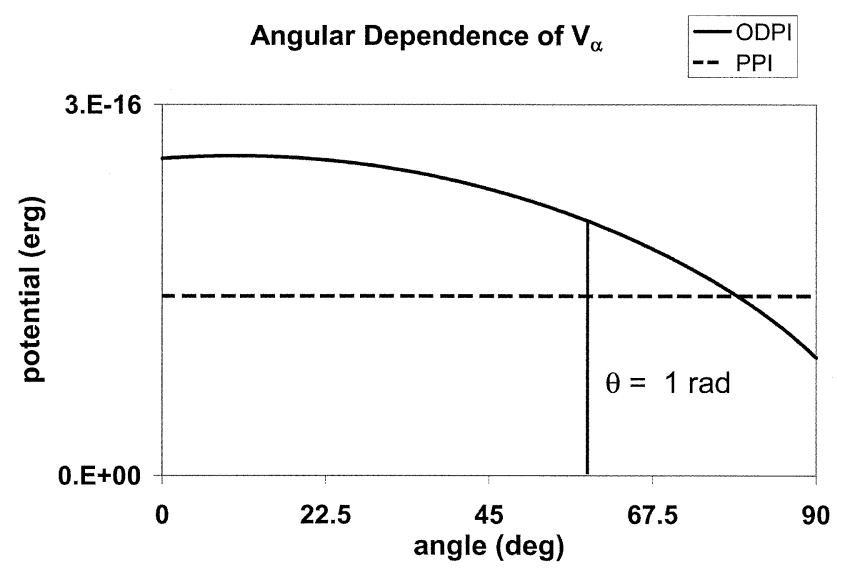

Figure 2. At a value of $\theta=1$ radian, the contribution of ODPI to the overall potential is greater than PPI. Data taken from an intermolecular distance of $10 \AA$. 
Table 2. Values of $\mathrm{k}_{\mathrm{L}}$ and $\mathrm{k}_{\mathrm{ODPI}}$ for the reaction of $\mathrm{C}_{n}^{-}$and $\mathrm{C}_{n} \mathrm{H}^{-}$with $\mathrm{O}$, and the percent deviation of $\mathrm{k}_{\mathrm{ODPI}}$ from $\mathrm{k}_{\mathrm{L}}$ and $\mathrm{k}_{\mathrm{PPI}}$.

\begin{tabular}{|c|c|c|c|c|}
\hline & $\mathrm{k}_{\mathrm{L}}$ & $\mathrm{k}_{\mathrm{ODPI}}$ & $\%$ deviation & $\%$ deviation \\
\hline & $\left(\mathrm{cm}^{3} / \mathrm{s}\right) \times 10^{-10}$ & $\left(\mathrm{~cm}^{3} / \mathrm{s}\right) \times 10^{-10}$ & (from Langevin) & (from PPI) \\
\hline $\mathrm{C}_{2}^{-}$ & 6.77 & 8.18 & 20.8 & 0.2 \\
\hline $\mathrm{C}_{4}^{-}$ & 6.05 & 7.39 & 22.1 & 2.8 \\
\hline $\mathrm{C}_{5}^{-}$ & 5.90 & 7.36 & 24.7 & 3.4 \\
\hline $\mathrm{C}_{6}^{-}$ & 5.80 & 7.34 & 26.6 & 2.7 \\
\hline $\mathrm{C}_{7}^{-}$ & 5.72 & 7.44 & 30.0 & 3.6 \\
\hline $\mathrm{C}_{8}^{-}$ & 5.66 & 7.53 & 33.0 & 3.9 \\
\hline $\mathrm{C}_{2} \mathrm{H}^{-}$ & 6.71 & 8.89 & 32.4 & 5.1 \\
\hline $\mathrm{C}_{4} \mathrm{H}^{-}$ & 6.04 & 7.97 & 32.0 & 2.8 \\
\hline $\mathrm{C}_{6} \mathrm{H}^{-}$ & 5.79 & 8.46 & 46.1 & 7.2 \\
\hline
\end{tabular}

at what $\alpha_{\mathrm{n}}$ does the ion-neutral dispersion interaction become significant? Two more common ions were evaluated, $\mathrm{OH}^{-}$and $\mathrm{H}_{3} \mathrm{O}^{+}$, and, using PPI, the value for $\alpha_{\mathrm{n}}$ below which deviation from $\mathrm{k}_{\mathrm{L}}$ becomes more than $5 \%$ was determined. For $\mathrm{OH}^{-}$, values of $\alpha_{\mathrm{n}}$ must be $<4.3 \AA^{3}$ and for $\mathrm{H}_{3} \mathrm{O}^{+}$, values must be $<1.0 \AA^{3}$ for the polarizability of the ion to be important. This clearly illustrates that the effect must be considered for most negative ions unless $\alpha_{\mathrm{n}}$ is quite high, whereas positive ions of similar size will only be affected by neutrals with very low polarizabilities (atomic species). For $\mathrm{C}_{6} \mathrm{H}^{-}$, the values of $\alpha_{\mathrm{n}}$ must be $<22.3 \AA^{3}$ for the polarizability of the ion to be important using PPI, and $<42.6 \AA^{3}$ when using ODPI. These relatively large values suggest that, for reactions of most negative ions, there is a strong dependence of the collision rate on the polarizability of the ion.

This calculation was also done for the benzene cation, whose larger analogs may have relevance to interstellar chemistry, specifically to the unidentified DIBs. In this case the polarizability of the ion is important when $\alpha_{\mathrm{n}}<9.5 \AA^{3}$ using PPI and when $\alpha_{\mathrm{n}}<26.9 \AA^{3}$ using ODPI. This result is quite interesting since collisions with $\mathrm{H}$ atom, for which $\alpha_{\mathrm{n}}=0.667 \AA^{3}$, are extremely important in the interstellar medium. An increase in the theoretical collision rates will have an impact on models of interstellar chemistry and physics.

\section{Conclusions}

While most ion-neutral reactions can be reasonably approximated as point charge systems, in reactions where either the polarizability of the ion is high or the polarizability of the neutral is low, the attractive potential between the two polarizable species must be considered. Ionic species in this category include most anions and large, $\pi$-system cations.

Implications of this consideration range from laboratory experiments to the interstellar medium. In the laboratory, many reactions that were previously thought to have efficiencies close to unity may have significantly lower efficiencies, indicating that new dynamic considerations may be warranted. Most models of the processes and interactions in the ISM use the Langevin theory to describe the collision frequency between ions and neutral molecules and atoms. In these environments, the significance of the induced dipoleinduced dipole adjustment may be substantial.

\section{Acknowledgments}

The authors gratefully acknowledge the National Aeronautics and Space Administration (Award NAG5-11487) for their financial support of this research. They thank Professors Stephen Leone, Robert Parson, and David Nesbitt and the reviewers for valuable comments and discussion.

\section{References}

1. Langevin, P. Ann. Chem. Phys. 1905, 5, 245.

2. Su, T.; Viggiano, A. A.; Paulson, J. F. The Effect of the Dipole-Induced Dipole Potential on Ion-Polar Molecule Collision Rate Constants. J. Chem. Phys. 1992, 96, 5550-5551.

3. Hsieh, E. T.; Castleman, A.W., Jr. A Reconsideration of the Theory of Capture Cross Sections for Ion/Molecule Reactions and a Total Energy and Angular Momentum Conserved Average Charge-Dipole Interaction Theory (TEAMS). Int. J. Mass Spectrom. Ion Phys. 1981, 40, 295-329.

4. Su, T.; Bowers, M. T. Ion-Polar Molecule Collisions. Effect of Ion Size on Ion-Polar Molecule Rate Constants. Parameterization of the Average-Dipole-Orientation Theory. Int. J. Mass Spectrom. Ion Phys. 1973, 12, 347-356.

5. Su, T.; Chesnavich, W. J. Parameterization of the Ion-Polar Molecule Collision Rate Constant by Trajectory Calculations. J. Chem. Phys. 1982, 76, 5183-5185.

6. Becke, A. D. Density-Functional Exchange-Energy Approximation with Correct Asymptotic Behavior. Phys. Rev. A 1988, 38, 3098-3100.

7. Lee, C.; Yang, W.; Parr, R. G. Development of the ColleSalvetti Correlation-Energy Formula into a Functional of the Electron Density. Phys. Rev. B 1988, 37, 785-789.

8. Miehlich, B.; Savin, A.; Stoll, H.; Preuss, H. Results Obtained with the Correlation Energy Density Functionals of Becke and Lee, Yang and Parr. Chem. Phys. Lett. 1989, 157, 200-206.

9. Su, T.; Su, E. C. F.; Bowers, M. T. Theory of Ion-Molecule Collisions: Effect of the Induced Dipole-Induced Dipole Potential on Ion-Molecule Capture Rate Constants. Int. J. Mass Spectrom. Ion Phys. 1978, 28, 285-288.

10. Hosseine, S.; Su, T. Effect of Induced Dipole-Induced Dipole Potential and the Size of Colliding Particles on Ion-Quadrupolar Molecule Collision Rate Constants. Iran J. Chem. Chem. Eng. 1998, 17, 42. 
11. Su, T.; Bowers, M. T. Classical Ion-Molecule Collision Theory. In Gas Phase Ion Chemistry; Bowers, M. T., Ed.; Academic Press Inc: New York, 1979; p 111.

12. Snow, T. P.; Le Page, V.; Keheyan, Y.; Bierbaum, V. M. The Interstellar Chemistry of PAH Cations. Nature 1998, 391, 259-260.

13. Le Page, V.; Keheyan, Y.; Snow, T. P.; Bierbaum, V. M. Gas Phase Chemistry of Pyrene and Related Cations with Molecules and Atoms of Interstellar Interest. Int. J. Mass Spectrom. 1999, 185/186/187, 949-959.

14. Le Page, V.; Keheyan, Y.; Snow, T. P.; Bierbaum, V. M.Reactions of Cations Derived from Naphthalene with Molecules and Atoms of Interstellar Interest. J. Am. Chem. Soc. 1999, 121(40), 9435-9446.

15. Le Page, V.; Snow, T. P.; Bierbaum, V. M. Hydrogenation and Charge States of PAHs in Diffuse Clouds. Part 1. Development of a Model. Ap. J. Suppl. Ser. 2001, 132(2), 233-251.

16. Le Page, V.; Snow, T. P.; Bierbaum, V. M. Hydrogenation and Charge States of PAHs in Diffuse Clouds. Part 2. Results. Ap. J. 2003, 584(1), 316-330.

17. Barckholtz, C.; Snow, T. P.; Bierbaum, V. M. Reactions of Cnand $\mathrm{CnH}-$ with Atomic and Molecular Hydrogen. Ap. J. 2001, 547(2), L171-L174. 